\title{
Erratum to: 1-Dependent Stationary Sequences for Some Given Joint Distributions of Two Consecutive Random Variables
}

\section{George Haiman}

Received: 30 October 2013 / Accepted: 19 December 2013 /

Published online: 12 January 2014

(C) Springer Science+Business Media New York 2014

\section{Erratum to: Methodol Comput Appl Probab (2012) 14:445-458 DOI 10.1007/s11009-011-9234-y}

p.446, in Proposition 2 "Suppose that a sequence of $\mathbb{N}^{n}$-valued functions $\left\{p_{n}\left(l_{1}, \ldots, l_{n}\right)\right\}_{n \geq 1}, \quad p_{n}\left(l_{1}, \ldots, l_{n}\right)=p\left(l_{1}, \ldots, l_{n}\right)$, satisfies the recurrence formula (3) for $n \geq 2$, where $p_{2}(i, j)=p(i, j)$ and $p_{1}(i)=p(i)$ are probability distributions which satisfy condition (1)" should be replaced by :"Suppose that a sequence of $\left(\mathbb{N}^{*}\right)^{n}$-valued functions $\left\{p_{n}\left(l_{1}, \ldots, l_{n}\right)\right\}_{n \geq 1}, p_{n}\left(l_{1}, \ldots, l_{n}\right)=p\left(l_{1}, \ldots, l_{n}\right)$, satisfies the recurrence formula (3) for $n \geq 2$, where $p_{2}(i, j)=p(i, j)$ and $p_{1}(i)=p(i)$ are probability distributions such that $p(i)=\sum_{j \in \mathbb{N}^{*}} p(i, j), \forall i \in \mathbb{N}^{*}$.

p.449, 1. 8 from bottom : "Then formula 3 ..." instead "Then formula 4..."

p.455, 1.1 : "Provided $A(m) \leq 1$ " should be replaced by " Provided $A(m) \leq .025$ "

The online version of the original article can be found at http://dx.doi.org/10.1007/s11009-011-9234-y.

G. Haiman $(\bowtie)$

Université de Lille 1, UFR de Mathématiques, Cité Scientifique,

59655 Villeneuve d'Ascq Cedex, France

e-mail: george.haiman@upmc.fr 